\title{
The association and dose-response relationship between dietary intake of $\alpha$-linolenic acid and risk of CHD: a systematic review and meta-analysis of cohort studies
}

\author{
Jingkai Wei ${ }^{1 *}$, Ruixue $\mathrm{Hou}^{2}$, Yuzhi $\mathrm{Xi}^{1}$, Alysse Kowalski ${ }^{3}$, Tiansheng Wang ${ }^{1}$, Zhi Yu ${ }^{4}$, Yirui Hu${ }^{5}$, \\ Eeshwar K. Chandrasekar ${ }^{6}$, Hao Sun ${ }^{7}$ and Mohammed K. Ali ${ }^{6,8}$ \\ ${ }^{1}$ Department of Epidemiology, Gilling School of Global Public Health, University of North Carolina at Chapel Hill, \\ 135 Dauer Drive, Chapel Hill, NC 27599, USA \\ ${ }^{2}$ Department of Nutrition, Gilling School of Global Public Health, University of North Carolina at Chapel Hill, \\ 135 Dauer Drive, Chapel Hill, NC 27599, USA \\ ${ }^{3}$ Nutrition and Health Science Doctoral Program, Laney Graduate School, Emory University, 1518 Clifton Road, Atlanta, \\ GA 30322, USA \\ ${ }^{4} J o h n s$ Hopkins Bloomberg School of Public Health, 615 North Wolfe Street, Baltimore, MD 21205, USA \\ ${ }^{5}$ Department of Biomedical and Translational Informatics, Geisinger Health System, 100 North Academy Avenue, Danville, \\ PA 17822, USA \\ ${ }^{6}$ School of Medicine, Emory University, 1648 Pierce Drive NE, Atlanta, GA 30307, USA \\ ${ }^{7}$ Department of Epidemiology, School of Public Health, China Medical University, 92 Bei'er Road, TaiYuan Jie ShangQuan, \\ Heping Qu, Shenyang Shi, Liaoning Sheng, 110003, People's Republic of China \\ ${ }^{8}$ Hubert Department of Global Health, Rollins School of Public Health, Emory University, 1518 Clifton Road, Atlanta, \\ GA 30322, USA
}

(Submitted 11 July 2017 - Final revision received 10 September 2017 - Accepted 26 October 2017)

\section{Abstract}

Previous studies show inconsistent associations between $\alpha$-linolenic acid (ALA) and risk of CHD. We aimed to examine an aggregate association between ALA intake and risk of CHD, and assess for any dose-response relationship. We searched the PubMed, EMBASE and Web of Science databases for prospective cohort studies examining associations between ALA intake and CHD, including composite CHD and fatal CHD. Data were pooled using random-effects meta-analysis models, comparing the highest category of ALA intake with the lowest across studies. Subgroup analysis was conducted based on study design, geographic region, age and sex. For dose-response analyses, we used twostage random-effects dose-response models. In all, fourteen studies of thirteen cohorts were identified and included in the meta-analysis. The pooled results showed that higher ALA intake was associated with modest reduced risk of composite CHD (risk ratios (RR) $=0.91 ; 95 \%$ CI $0 \cdot 85,0.97)$ and fatal CHD $(\mathrm{RR}=0.85 ; 95 \%$ CI $0.75,0.96)$. The analysis showed a J-shaped relationship between ALA intake and relative risk of composite CHD $\left(\chi^{2}=21.95, P<0 \cdot 001\right)$. Compared with people without ALA intake, only people with ALA intake $<1.4 \mathrm{~g} / \mathrm{d}$ showed reduced risk of composite CHD. ALA intake was linearly associated with fatal CHD - every $1 \mathrm{~g} / \mathrm{d}$ increase in ALA intake was associated with a $12 \%$ decrease in fatal CHD risk (95\% CI -0.21, -0.04). Though a higher dietary ALA intake was associated with reduced risk of composite and fatal $\mathrm{CHD}$, the excess composite CHD risk at higher ALA intakes warrants further investigation, especially through randomised controlled trials.

Key words: $\alpha$-Linolenic acid: CHD: Meta-analyses: Dose-response relationships

According to the WHO, IHD or CHD accounted for 7.4 million deaths in 2012, the leading cause of death worldwide ${ }^{(1,2)}$. There is growing evidence showing that long-chain $n$-3 PUFA, particularly EPA (C20:5n-3) and DHA (C22:6n-3), are beneficial for prevention of $\mathrm{CVD}^{(3)}$. The American Heart Association recommends two servings of fatty fish per week or $500 \mathrm{mg}$ EPA and DHA per day be taken for those without $\mathrm{CHD}$, and $1 \mathrm{~g} / \mathrm{d}$ of EPA/DHA for those with
$\mathrm{CHD}^{(4)}$. The main source of EPA and DHA is fish ${ }^{(5)}$. However, the increasing demand on fish consumption may cause problems of sustainability in provision of fish, and pollution - specifically, the accumulation of toxic materials - may neutralise the health benefits of fish, or even have adverse effects ${ }^{(6)}$. Also, fish is not applicable for most vegetarians. Therefore, alternative sources of EPA and DHA are needed to maintain the level of intake.

Abbreviations: ALA, $\alpha$-linolenic acid; RR, risk ratio.

* Corresponding author: J. Wei, email jingkai@live.unc.edu 
$\alpha$-Linolenic acids (ALA), an $n-3$ fatty acid mainly derived from soyabean, flaxseed and walnuts has been proposed as a potential substitution for fish. ALA is converted to long-chain $n-3$ fatty acids in the body, including EPA and DHA, though the efficiency is low ${ }^{(7)}$. A number of studies have been conducted to examine associations between ALA intake and CHD risk ${ }^{(8)}$, though the results were inconsistent.

A previous meta-analysis of Pan et al ${ }^{(9)}$ indicates that a higher level of ALA intake is associated with reduced risk of fatal CHD, whereas not associated with risk of non-fatal CHD or composite CHD. It also indicates that each additional $1 \mathrm{~g} / \mathrm{d}$ ALA intake is associated with a $10 \%$ reduction in risk of fatal CHD. Recently, several new studies have examined the associations between ALA intake and risk of $\mathrm{CHD}^{(10-14)}$, so an updated aggregate association may be informative. Moreover, it will be beneficial to explore the optimal dosing of ALA intake for prevention of CHD - specifically, what threshold of intake is needed for benefit and whether higher doses confer additional benefit. We systematically reviewed the literature and conducted a meta-analysis to investigate the potential dose-response relationship between ALA intake and CHD risk. We hypothesised that higher intake of ALA is linearly associated with lower risk of composite and fatal CHD.

\section{Methods}

\section{Search strategy and eligibility criteria}

We systematically searched the PubMed, Embase and Web of Science databases for epidemiological studies published in peer-reviewed journals over a 50-year period (from January 1966 to August 2017) that investigated the associations between dietary ALA intake and CHD. Search terms included 'alphalinolenic acid', 'cardiovascular disease', 'coronary heart disease', 'coronary artery disease', 'ischemic heart disease' and 'myocardial infarction' (see the online Supplementary Appendix). We also searched for studies listed or cited in review papers, in case there were potential studies not captured by the database search strategy. The search was limited to articles written in English. We contacted authors of included articles if important information was missing.

We included original full-text studies that were: (1) cohort studies, (2) included adult participants free from CHD history with assessments of dietary intake of ALA, (3) assessed primary outcomes including fatal and/or non-fatal CHD and (4) had a comparison between the highest and the lowest level of dietary ALA intake. We excluded studies that: (1) included only assessed ALA level in plasma or serum as biomarkers, and (2) included composite outcomes that combined different cardiovascular events and CHD could not be distinguished. If there were several studies based on the same cohort, we retained the studies with the most directly relevant results for particular outcomes.

Study selection was conducted in three steps. First, the titles of studies identified in our literature search were independently reviewed by three reviewers (J. W., R. H., Y. X.). Second, the abstracts of studies that remained after the initial screening were reviewed by three reviewers and disagreements were reconciled. Third, data from studies that met inclusion criteria were extracted, including sample size, study design, age, sex, methods of assessing dietary intake of ALA, amount of ALA intake, outcome of studies and covariates included in the analysis.

\section{Definitions}

The information regarding dietary ALA intake was obtained from validated tools, such as FFQ, 24-h dietary recalls, dietary history and food records. The outcomes of our proposed metaanalysis were CHD events defined as one of the following terms: coronary heart disease, coronary artery disease, ischemic heart disease, or myocardial infarction. Composite CHD was defined as total incident cases of CHD events reported in the studies, including fatal or non-fatal CHD. The definition of fatal CHD included CHD death or CHD mortality mentioned in the articles. If a study reported only fatal $\mathrm{CHD}$ or non-fatal $\mathrm{CHD}$, then it would be also counted into composite CHD. All incident cases were ascertained by physician diagnosis, medical records or death certificate.

\section{Quality assessment}

Quality assessment was conducted using the Newcastle-Ottawa Scale ${ }^{(15)}$. The Newcastle-Ottawa Scale evaluates quality of cohort studies in three domains:

(1) selection of exposed and non-exposed cohorts (representativeness of the exposed cohort, selection of the non-exposed cohort, ascertainment of exposure, and demonstration of absence of outcome at the beginning of studies);

(2) comparability of exposed and non-exposed cohorts (analysis appropriately adjusted for potential confounding factors, including the most important and additional factors, such as medications, history of other chronic diseases and lifestyle factors); and

(3) outcome ascertainment (adequacy of outcome assessment, length of follow-up and adequacy of follow-up).

A study would be awarded a maximum of 1 point for each variable within each assessment domain (selection, comparability, and outcome) for a possible maximum total score of 9 . The quality assessment was conducted independently by two reviewers (R. H. and A. K.), and the results were reconciled until a consensus was reached.

\section{Statistical analysis}

We obtained fully adjusted risk ratios (RR) and 95\% CI from each study. We pooled data across studies using random-effects meta-analysis models, and weighted these by the inverse of the estimated variance, comparing the highest category of ALA intake with the lowest as indicated in each study. We considered the pooled RR to be statistically significant if 95\% CI did not contain 1 . We generated forest plots to illustrate individual and pooled risk estimates, and funnel plots to examine potential publication bias. We calculated the $I^{2}$ statistic to quantify the proportion of between-study heterogeneity attributable to variability in the association rather than sampling variation. We also conducted subgroup analyses to examine for heterogeneity of associations by regions where studies were conducted (North America, Europe, Asia), mean age of study participants ( $\geq 60$ years or not), and percentage of female subjects ( $\geq 50 \%$ or not). 
To examine impacts of the amount of ALA intake and risk of CHD, we conducted a dose-response meta-analysis for each outcome. For each study, we assigned the median or mean grams of ALA for each exposure level to the corresponding RR. To explore potential linear and non-linear relationships between ALA intake and CHD risk, we performed randomeffects dose-response meta-analyses. We estimated the predicted relative risk by comparing specific levels of ALA intake with no ALA intake based on the linear model or spline transformation, as appropriate. The linear or non-linear relationship between dietary ALA intake and CHD risk was considered significant if the $\mathrm{p}$ value of the chi-square statistic was smaller than $0 \cdot 05$.

All analyses were conducted using Stata 14 (StataCorp).

\section{Results}

\section{Study characteristics}

The database search started with a total of 1853 citations (Fig. 1). We identified an additional twelve studies from the bibliographies of relevant reports and reviews. After eliminating duplicates, 1422 remained. Of these, we excluded 1368 studies due to irrelevant topics, non-prospective designs, or assessments of exposures and outcomes not meeting the inclusion criteria. We retrieved full-texts of the fifty-four remaining articles to be examined in more detail. Of these, fourteen prospective studies eventually met all inclusion criteria and were included in the systematic review and meta-analysis.

The online Supplementary Table S1 showed characteristics of included studies. The pooled analysis included data from 345202 individuals, with a mean length of follow-up between 4 and 22 years. The mean (median) ages of participants ranged from 41.5 to 73.0 years. Included studies were conducted in the USA, the Netherlands, Finland, Denmark, Spain and Singapore. Two studies stratified the analysis into male and female participants separately ${ }^{(14,16)}$. Two studies were based on the same cohort (one is used for meta-analysis, and one for dose-response analysis). Nine studies examined associations between dietary ALA intake and risk of composite $\mathrm{CHD}^{(10,11,14,16-22)}$. Eight studies examined fatal $\mathrm{CHD}$ as outcomes $^{(11-13,17-19,23,24)}$.

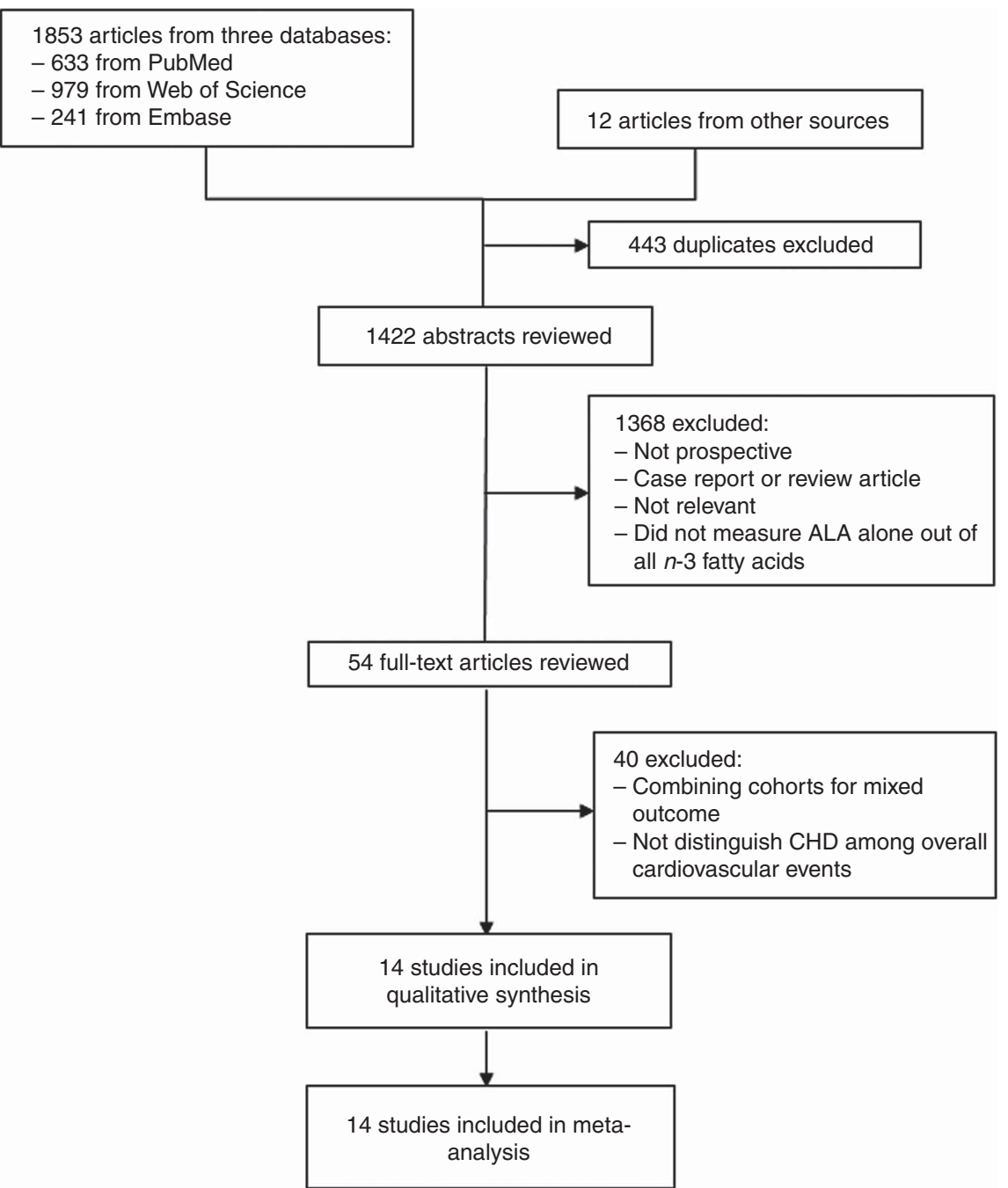

Fig. 1. Flow chart showing selection of study reports for the meta-analysis. ALA, a-linolenic acid. 


\section{Pooled analyses}

The meta-analysis of fourteen groups of analysis across twelve studies showed that higher intake of ALA was moderately associated with reduced risk of composite CHD (Fig. 2(a), pooled
( $\mathrm{RR}=0.91 ; 95 \% \mathrm{CI} 0 \cdot 85,0 \cdot 97)$. The analysis of nine studies indicated that a higher dietary ALA intake was associated with a lower risk of fatal CHD (Fig. 2(b), pooled RR=0.85; 95\% CI 0.75, 0.96). Heterogeneity was not detected in the associations with ALA intake with risk of composite $\mathrm{CHD}\left(I^{2}=7.9 \%, P=0.36\right)$, or fatal $\mathrm{CHD}$

(a)

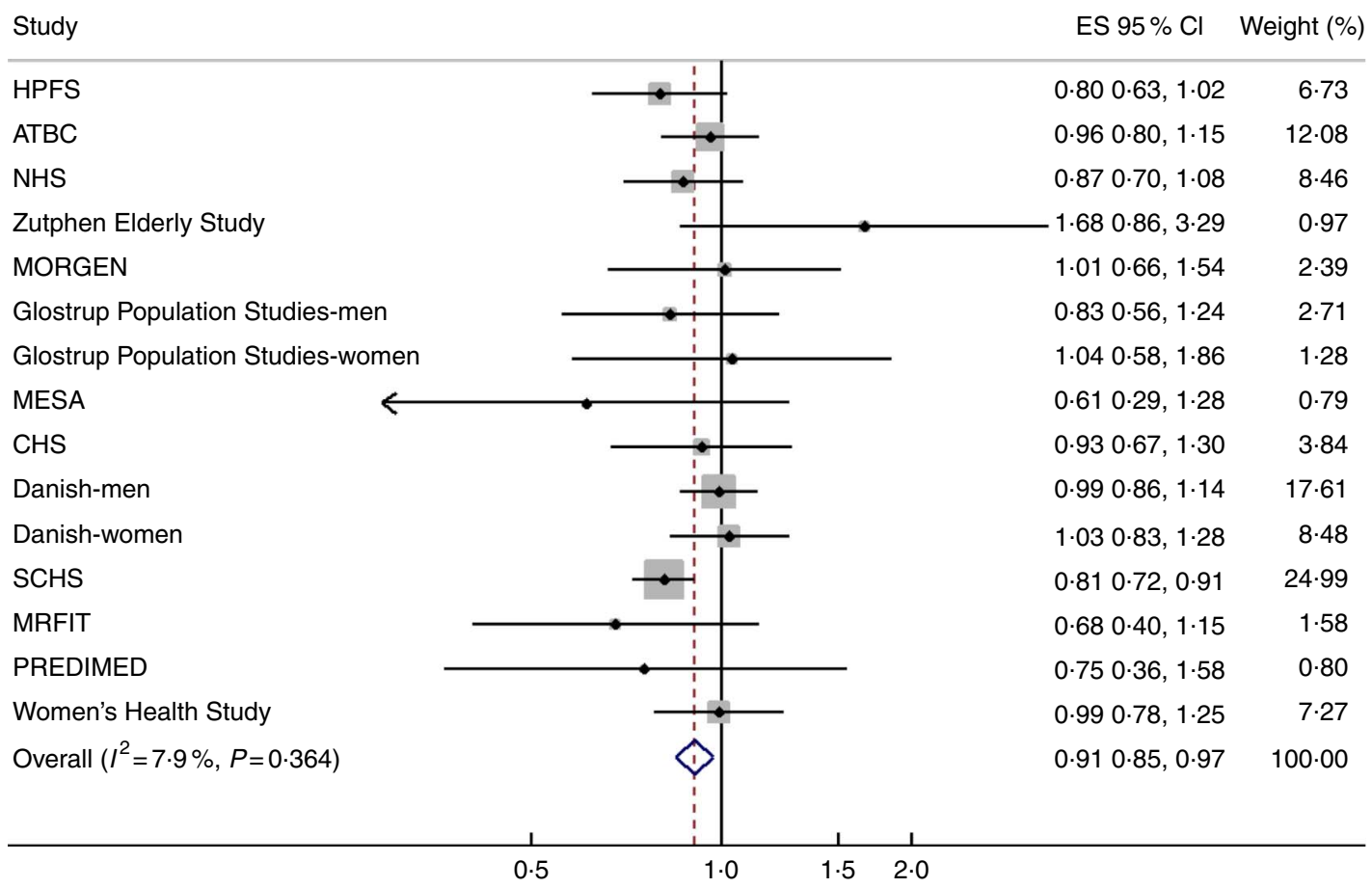

(b)

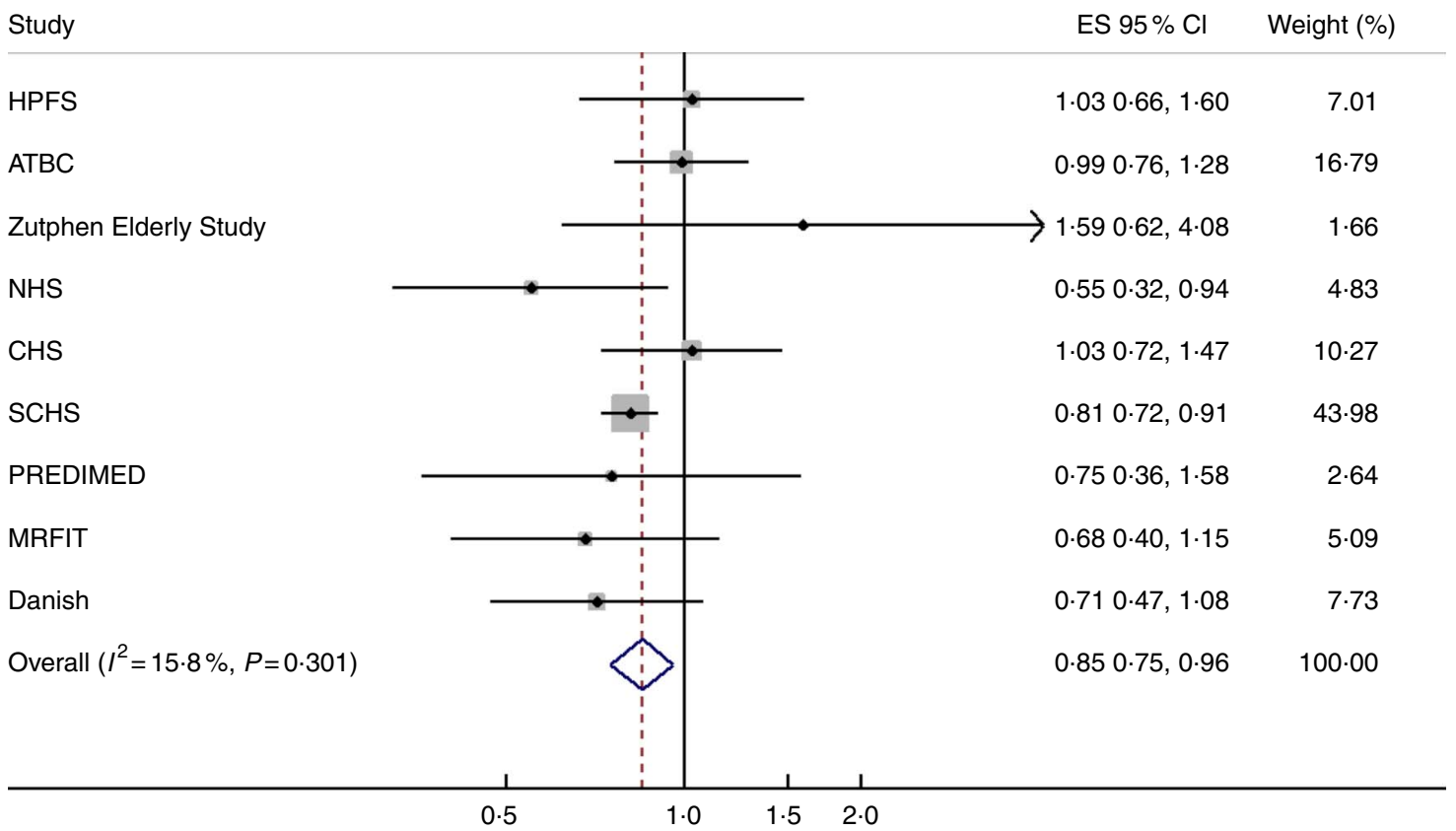

Fig. 2. Pooled association between dietary a-linolenic acid (ALA) intake and risk of (a) composite CHD and (b) fatal CHD. Weights are from random-effects analysis. ES, effect size; HPFS, Health Professionals Follow-Up Study; ATBC, Alpha-Tocopherol, Beta-Carotene Cancer Prevention; NHS, Nurses' Health Study; MORGEN, Monitoring Project on Risk Factors and Chronic Diseases in the Netherlands; MESA, Multi-Ethnic Study of Atherosclerosis; CHS, Cardiovascular Health Study; SCHS, Singapore Chinese Health Study; MRFIT, Multiple Risk Factor Intervention Trial; PREDIMED, Prevención con Dieta Mediterránea. 
Table 1. Subgroup meta-analysis of the pooled analysis between dietary a-linolenic acid (ALA) intake and risk of CHD (Pooled risk ratios (RR) and $95 \%$ confidence intervals)

\begin{tabular}{|c|c|c|c|c|c|c|c|c|}
\hline & \multicolumn{3}{|c|}{ Composite CHD (n 12) } & \multirow[b]{2}{*}{$P(\%)^{*}$} & \multicolumn{4}{|c|}{ Fatal CHD $(n 9)$} \\
\hline & Number of studies & Pooled RR & $95 \% \mathrm{Cl}$ & & Number of studies & Pooled RR & $95 \% \mathrm{Cl}$ & $P(\%)$ \\
\hline \multicolumn{9}{|l|}{ Regions } \\
\hline North America & 6 & 0.88 & $0.80,0.98$ & 0 & 4 & 0.83 & $0.62,1.12$ & 40.8 \\
\hline Europe & 6 & 0.99 & $0.90,1.08$ & 0 & 4 & 0.91 & $0.72,1.15$ & 11.0 \\
\hline Asia & 1 & 0.81 & $0.72,0.90$ & NA & 1 & 0.81 & $0.72,0.90$ & NA \\
\hline \multicolumn{9}{|l|}{ Mean age } \\
\hline$\geq 60$ years & 4 & 0.94 & $0.66,1.34$ & 33.8 & 3 & 1.02 & $0.76,1.38$ & 0.0 \\
\hline$<60$ years & 9 & 0.90 & $0.85,0.96$ & $4 \cdot 1$ & 6 & 0.82 & $0.72,0.94$ & 18.7 \\
\hline \multicolumn{9}{|l|}{ Female $\dagger$} \\
\hline$\geq 50 \%$ & 9 & 0.88 & $0.82,0.95$ & 0.0 & 4 & 0.81 & $0.68,0.97$ & 20.8 \\
\hline$<50 \%$ & 6 & 0.93 & $0.82,1.05$ & $28 \cdot 1$ & 5 & 0.90 & $0.74,1.11$ & $13 \cdot 2$ \\
\hline
\end{tabular}

RR, risk ratio; NA, not applicable.

* $P>0.05$ for all $I^{2}$.

† For composite $\mathrm{CHD}$, two studies were analysed by sex, so they were included in both categories.

$\left(I^{2}=15 \cdot 8 \%, P=0 \cdot 30\right)$. There was no detected publication bias across studies, as the funnel plots showed good symmetry (online Supplementary Fig. S1 and S2).

Results of subgroup analyses showed that a higher ALA intake was associated with risk reductions of composite CHD in studies conducted in North America and Asia, studies with a lower percentage of female participants $(<50 \%)$ and studies with lower mean age ( $<60$ years). For fatal CHD, subgroup analyses showed risk reductions with ALA intake in studies conducted in Asia, studies with more female ( $\geq 50 \%)$ and younger (<60 years) participants (Table 1 ).

The two-stage random-effects dose-response analysis did not show a linear relationship between dietary ALA intake and relative risk of composite $\mathrm{CHD}\left(\chi^{2}=1 \cdot 13, P=0 \cdot 29\right)$, but rather a $\mathrm{J}$-shaped curve between ALA intake and relative risk of composite CHD $\left(\chi^{2}=21.95, P<0 \cdot 001\right)$. ALA intake $<1.4 \mathrm{~g} / \mathrm{d}$ showed reduced risk of composite $\mathrm{CHD}$, compared with people without ALA intake. A quantity of $1 \mathrm{~g}$ of ALA intake per day was associated with the lowest risk of CHD (Fig. 3(a)). There was a linear relationship between ALA intake and risk of fatal CHD. ALA intake, $1 \mathrm{~g} / \mathrm{d}$, was associated with $12 \%$ decrease in risk of fatal CHD $(\beta=-0 \cdot 12 ; 95 \%$ CI $-0 \cdot 21,-0 \cdot 04)$ (Fig. 3(b)).

\section{Quality assessment}

The quality of the fourteen studies included in the meta analysis, as scored with the Newcastle-Ottawa scale, is presented in the online Supplementary Table S2. The mean total quality score was 7.4 out of a maximum score of 9 (range 7-9) indicating that, overall, the methodological quality among cohort studies was moderately good.

\section{Discussion}

The present systematic review and meta-analysis showed significant associations between a higher ALA intake with reduced risk of composite $\mathrm{CHD}$ and fatal $\mathrm{CHD}$. Our doseresponse analysis showed that compared with people without intake of ALA, only a certain range of ALA intake $(<1.4 \mathrm{~g} / \mathrm{d})$ was associated with reduced risk of CHD. In contrast, a higher
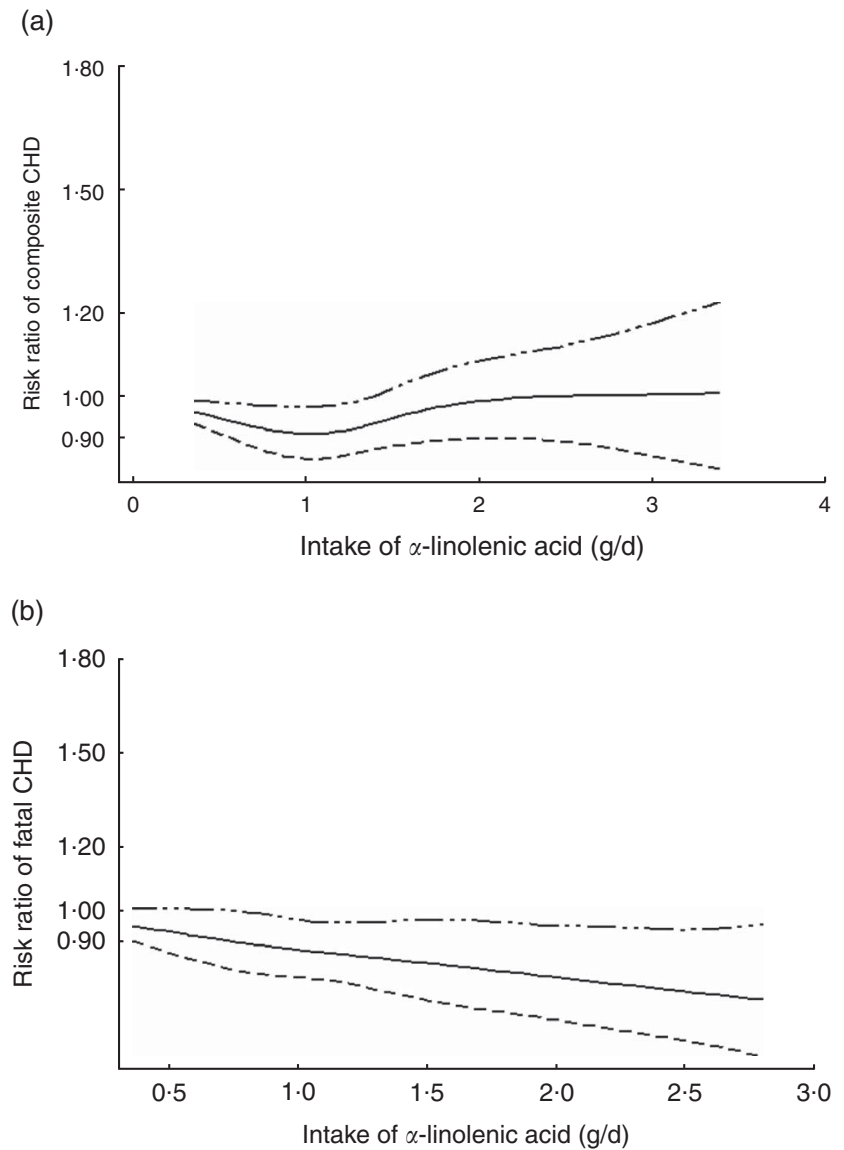

Fig. 3. Dose-response relationship between a-linolenic acid (ALA) intake and relative risk of (a) composite $\mathrm{CHD}$ and (b) fatal $\mathrm{CHD}$. Weights are from randomeffects analysis. - - - - Lower limit of $95 \% \mathrm{Cl}$; - . - , upper limit of $95 \% \mathrm{Cl}$; , risk ratio.

intake of ALA was associated with reduced risk of fatal CHD. To our knowledge, this is the largest meta-analysis of dietary ALA intake and CHD risk based on prospective studies. However, given the present publication bias and heterogeneity among studies, the results should be interpreted with caution. 
Our results were similar with previous studies to some extent. Pan et al. ${ }^{(9)}$ showed a significant inverse association and a linear dose-response relationship between ALA intake and risk of fatal CHD, though it did not show an overall CHD outcome, but a composite of all cardiovascular events, including CHD, stroke and other CVD events with an association of similar magnitude. Vedtofte et al. ${ }^{(25)}$ previously found that $1 \mathrm{~g} / \mathrm{d}$ of ALA consumed was associated with a $15 \%$ lower risk of CHD events and a $23 \%$ lower risk of CHD deaths in males. Although it did not reach statistical significance, a potential non-linear relationship was not tested. Our dose-response analysis indicated a non-linear relationship between ALA intake and risk of composite CHD, which suggested a beneficial level of dietary ALA intake for prevention of CHD. The National Academy of Medicine recommended that men should consume $1.6 \mathrm{~g}$ of ALA per day and women should consume $1.1 \mathrm{~g} / \mathrm{d}^{(26)}$. This is similar to our present result of optimal level of ALA $(1 \mathrm{~g} / \mathrm{d})$ in the doseresponse meta-analysis.

Although ALA shows promising benefits in CHD prevention, the effect seems to be smaller than expected, particularly our dose-response analysis showed a non-linear relationship. This may be explained by residual or unknown confounding effects, such as intake of other fatty acids or dietary components related to risk of CHD. Furthermore, as Oomen et al. ${ }^{(19)}$ point out, that intake of ALA may be associated with intake of trans-fatty acids, which is associated with an increased risk of $\mathrm{CHD}^{(19)}$. ALA and trans-fatty acids are also found correlated in biomarkers ${ }^{(27)}$. According to our present results, it is likely that under a certain amount of ALA intake, ALA has an effect over trans-fatty acids, whereas trans-fatty acids may play a more important role in association with CHD. However, this does not affect an expected trend of risk in fatal CHD.

The mechanisms of association between dietary ALA intake and CHD are not fully understood yet. The conversion from ALA to EPA and DHA may partially account for the association, whereas previous studies have found that only very limited amounts of ALA were actually converted ${ }^{(28)}$, so the efficiency is not high. Currently, there is no solid evidence showing a direct causal relationship between dietary ALA intake and CHD risk, whereas previous epidemiological studies showed some clues, for example, Lemaitre et al. ${ }^{(29)}$ found an association between ALA in erythrocytes and risks of sudden cardiac death, which is independent of erythrocyte levels of EPA and DHA, linoleic acid and trans-fatty acids. There are more plausible mechanisms to account for the association between ALA intake and CHD, such as reduced inflammation, which is found to be associated with higher intake of nuts ${ }^{(30)}$. Potential biological research is needed to further elucidate the mechanisms involved.

Our findings have significant relevance for public health and medicine. A number of studies also examined the association of biomarkers of ALA and CHD risk, whereas studies may have a better significance in guidance of human diet with dietary ALA intake as an exposure Diet has been recognised as an essential aspect in preventing CHD. In commonly applied dietary patterns for prevention of CVD, such as the Mediterranean dietary pattern $^{(31)}$ and Dietary Approaches to Stop Hypertension ${ }^{(32)}$, both emphasize intake of fish, which is the main source of EPA and DHA. As an alternative source of EPA and DHA, ALA should be considered as an important element in CHD prevention, as the potential for supplying ALA is great ${ }^{(5)}$. It is expected that a guideline for specific groups of foods and amount be made to the public for self-administered prevention of CHD. Furthermore, ALA could potentially lead to pharmacological applications. Randomised controlled trials on CHD patients have shown that ALA intake may reduce risk of CHD events, including recurrence of $\mathrm{CHD}$ and $\mathrm{CHD}$ death ${ }^{(33,34)}$. Future randomised controlled trials are needed to examine the effects of ALA on reducing primary CHD risk.

Our present study had some noteworthy limitations. First, all the included studies did not report uniform levels of dietary ALA intake and did not report the same outcomes, although the results were consistent in general. Second, our present metaanalysis of prospective cohort studies only included two randomised controlled trials. More randomised controlled trials of the effect of ALA intake on reduction of CHD are needed to remove all unmeasured confounding that may still plague prospective observational studies.

In conclusion, existing prospective studies suggest that higher ALA intake is associated with reduced risk of composite CHD and fatal CHD. Though a higher dietary ALA intake was associated with reduced risk of fatal CHD, the excess composite CHD risk at higher ALA intakes warrants further investigation, especially through randomised controlled trials.

\section{Acknowledgements}

The authors thank authors of the included articles for additional information relevant to the analysis.

This research received no specific grant from any funding agency, commercial or not-for-profit sectors.

J. W. designed the study. J. W., R. H. and Y. X. conducted the literature search and data extraction. R. H. and A. K. performed quality assessment. J. W. conducted data analysis and drafted the manuscript. All authors contributed to and approved the final manuscript.

None of the authors has any conflicts of interest to declare.

\section{Supplementary material}

For supplementary material/s referred to in this article, please visit https://doi.org/10.1017/S0007114517003294

\section{References}

1. Mathers CD, Fat DM, Inoue M, et al. (2005) Counting the dead and what they died from: an assessment of the global status of cause of death data. Bull World Health Organ 83, 171-177.

2. Al-Mawali A (2015) Non-communicable diseases: shining a light on cardiovascular disease, Oman's biggest killer. Oman Med J 30, 227-228.

3. Sala-Vila A, Estruch R \& Ros E (2015) New insights into the role of nutrition in CVD prevention. Curr Cardiol Rep 17, 26.

4. Kris-Etherton PM, Harris WS, Appel LJ, et al. (2002) Fish consumption, fish oil, omega-3 fatty acids, and cardiovascular disease. Circulation 106, 2747-2757. 
5. Mozaffarian D \& Wu JHY (2011) Omega-3 fatty acids and cardiovascular disease: effects on risk factors, molecular pathways, and clinical events. J Am Coll Cardiol 58, 2047-2067.

6. Mozaffarian D, Appel LJ \& Van Horn L (2011) Components of a cardioprotective diet: new insights. Circulation 123, 2870-2891.

7. Rajaram S (2014) Health benefits of plant-derived alphalinolenic acid. Am J Clin Nutr 100, Suppl. 1, 443s-448s.

8. Mozaffarian D (2005) Does alpha-linolenic acid intake reduce the risk of coronary heart disease? A review of the evidence. Altern Ther Health Med 11, 24-30; quiz 31, 79.

9. Pan A, Chen M, Chowdhury R, et al. (2012) Alpha-linolenic acid intake and biomarker in relation to risk of cardiovascular disease: a meta-analysis and systematic review. Am J Clin Nutr 96, 1262-1273.

10. Otto MCD, Wu JHY, Baylin A, et al. (2013) Circulating and dietary omega- 3 and omega- 6 polyunsaturated fatty acids and incidence of CVD in the Multi-Ethnic Study of Atherosclerosis. I Am Heart Assoc 2, e000506.

11. Fretts AM, Mozaffarian D, Siscovick DS, et al. (2014) Plasma phospholipid and dietary alpha-linolenic acid, mortality, CHD and stroke: the Cardiovascular Health Study. Br J Nutr 112, 1206-1213.

12. Koh AS, Pan A, Wang R, et al. (2015) The association between dietary omega-3 fatty acids and cardiovascular death: the Singapore Chinese Health Study. Eur J Prev Cardiol 22 364-372

13. Sala-Vila A, Guasch-Ferre M, Hu FB, et al. (2016) Dietary alpha-linolenic acid, marine omega-3 fatty acids, and mortality in a population with high fish consumption: findings from the PREvencion con DIeta MEDiterranea (PREDIMED) Study. J Am Heart Assoc 5, e002543.

14. Bork CS, Jakobsen MU, Lundbye-Christensen S, et al. (2016) Dietary intake and adipose tissue content of alpha-linolenic acid and risk of myocardial infarction: a Danish cohort study. Am J Clin Nutr 104, 41-48.

15. Wells GA, Shea B, O'Connell D, et al. (2000) The NewcastleOttawa Scale (NOS) for assessing the quality of nonrandomised studies in meta-analyses. http://www.ohri.ca/ programs/clinical_epidemiology/oxford.asp (accessed November 2017).

16. Vedtofte MS, Jakobsen MU, Lauritzen L, et al. (2011) Dietary alpha-linolenic acid, linoleic acid, and n-3 long-chain PUFA and risk of ischemic heart disease. Am J Clin Nutr 94 1097-1103.

17. Ascherio A, Rimm EB, Giovannucci EL, et al. (1996) Dietary fat and risk of coronary heart disease in men: cohort follow up study in the United States. BMJ 313, 84-90.

18. Pietinen $\mathrm{P}$, Ascherio A, Korhonen $\mathrm{P}$, et al. (1997) Intake of fatty acids and risk of coronary heart disease in a cohort of Finnish men. The Alpha-Tocopherol, Beta-Carotene Cancer Prevention Study. Am J Epidemiol 145, 876-887.

19. Oomen CM, Ocke MC, Feskens EJ, et al. (2001) alphaLinolenic acid intake is not beneficially associated with $10-\mathrm{y}$ risk of coronary artery disease incidence: the Zutphen Elderly Study. Am J Clin Nutr 74, 457-463.

20. de Goede J, Verschuren WM, Boer JM, et al. (2011) Alphalinolenic acid intake and 10-year incidence of coronary heart disease and stroke in 20,000 middle-aged men and women in the Netherlands. PLOS ONE 6, e17967.

21. Albert CM, Oh K, Whang W, et al. (2005) Dietary alpha-linolenic acid intake and risk of sudden cardiac death and coronary heart disease. Circulation $\mathbf{1 1 2}$, 3232-3238.

22. Rhee JJ, Kim E, Buring JE, et al. (2017) Fish consumption, omega-3 fatty acids, and risk of cardiovascular disease. Am J Prev Med 52, 10-19.

23. Dolecek TA (1992) Epidemiological evidence of relationships between dietary polyunsaturated fatty acids and mortality in the multiple risk factor intervention trial. Proc Soc Exp Biol Med 200, 177-182.

24. Hu FB, Stampfer MJ, Manson JE, et al. (1999) Dietary intake of alpha-linolenic acid and risk of fatal ischemic heart disease among women. Am J Clin Nutr 69, 890-897.

25. Vedtofte MS, Jakobsen MU, Lauritzen L, et al. (2014) Association between the intake of alpha-linolenic acid and the risk of CHD. Br J Nutr 112, 735-743.

26. Zatonski W, Campos H \& Willett W (2008) Rapid declines in coronary heart disease mortality in Eastern Europe are associated with increased consumption of oils rich in alphalinolenic acid. Eur J Epidemiol 23, 3-10.

27. Pedersen JI, Ringstad J, Almendingen K, et al. (2000) Adipose tissue fatty acids and risk of myocardial infarction - a case-control study. Eur J Clin Nutr 54, 618-625.

28. Barcelo-Coblijn G \& Murphy EJ (2009) Alpha-linolenic acid and its conversion to longer chain $n-3$ fatty acids: benefits for human health and a role in maintaining tissue $n-3$ fatty acid levels. Prog Lipid Res 48, 355-374.

29. Lemaitre RN, King IB, Sotoodehnia N, et al. (2009) Red blood cell membrane alpha-linolenic acid and the risk of sudden cardiac arrest. Metabolism 58, 534-540.

30. Yu Z, Malik VS, Keum N, et al. (2016) Associations between nut consumption and inflammatory biomarkers. Am J Clin Nutr 104, 722-728.

31. Sanchez-Villegas A, Martinez JA, De Irala J, et al. (2002) Determinants of the adherence to an 'a priori' defined Mediterranean dietary pattern. Eur J Nutr 41, 249-257.

32. Sacks FM, Obarzanek E, Windhauser MM, et al. (1995) Rationale and design of the Dietary Approaches to Stop Hypertension trial (DASH). A multicenter controlled-feeding study of dietary patterns to lower blood pressure. Ann Epidemiol 5, 108-118.

33. de Lorgeril M, Renaud S, Mamelle N, et al. (1994) Mediterranean alpha-linolenic acid-rich diet in secondary prevention of coronary heart disease. Lancet 343, 1454-1459.

34. Kromhout D, Giltay EJ \& Geleijnse JM (2010) n-3 fatty acids and cardiovascular events after myocardial infarction. $N$ Engl J Med 363, 2015-2026. 